\title{
INTRODUÇÃO À GESTÃO DE CONTEÚDOS
}

\author{
Júlio C. I. Pereira
}

Curso de Ciência da Computação, Faculdade de Pedro Leopoldo

Marcello P. Bax

Escola de Ciência da Informação, Universidade Federal de Minas

Gerais (UFMG)

Através de uma compilação de vários trabalhos relacionados, o artigo descreve a Gestão de Conteúdos em ambiente web, caracteriza os sistemas de Gestão de Conteúdos e apresenta as principais ferramentas disponíveis, assim como os benefícios e os riscos associados à sua implantação. Para fixar as idéias, levanta-se elementos para justificar o investimento financeiro em uma solução de Gestão de Conteúdos. Após introduzir o tema, propõe uma diferenciação clara entre informação, conhecimento e conteúdo, e sugere que a Gestão de Conteúdos permite a Gestão de Conhecimento na prática. Caracteriza as ferramentas de Gestão de Conteúdos denominadas CMS e apresenta vantagens e benefícios advindos de sua implementação nas organizações. Apresenta uma avaliação que permite determinar os retornos sobre investimentos nesse tipo de tecnologia. $\mathrm{O}$ artigo parte do pressuposto de que para acompanhar a evolução da Internet como um canal de comunicação interno e externo - de relacionamento com clientes, fornecedores e parceiros - as empresas precisam investir em tecnologias que permitem a distribuição e a publicação de informações de forma ágil, segura e confiável. Inerente à essa evolução, vem a sofisticação das informações que devem estar online, em páginas web com imagens, filmes, figuras etc. Alterando a forma de se criar e manter páginas web, estão sendo criadas ferramentas conhecidas como CMS Content Management System's - que conferem a agilidade, segurança e a confiabilidade requeridas para o tratamento da informação em um novo estágio da Internet. As ferramentas CMS incluem recursos que permitem o gerenciamento de conteúdos, vindos de todas as fontes e formas possíveis, com múltiplos esquemas de tratamento e fluxos de trabalho, podendo ser customizados e integrados, facilitando o uso de novos mecanismos de consultas e permitindo publicações nos mais diferenciados dispositivos. Os benefícios ligados a adoção de um CMS incluem desde a redução do custo de atualização dos conteúdos nos websites até o aumento da eficiência das equipes de TI. Como contribuição o artigo descreve benefícios ligados a adoção de um CMS pelas empresas que incluem desde a redução de custo de manutenção de websites até um aumento dos canais de publicação dos conteúdos, trazendo aumento de clientes e de negócios. Apresenta também os desafios associados que são a definição e padronização de regras para organizar informações em "repositórios" e a necessidade de se disponibilizar esse conteúdo informacional de forma padronizada, mas customizada para cada cliente e tipo de canal de comunicação. Considerando os benefícios advindos da possibilidade de criação, distribuição e a publicação de conteúdos de forma mais ágil, mais barata e confiável, espera-se que a Gestão de Conteúdos seja implementada nas corporações nos próximos anos através da adoção de alguma ferramenta CMS e de uma mudança comportamental que torne possível o uso desses novos recursos.

Palavras-chave: Portais Corporativos, Gestão de Conteúdos, Content Management, Gestão de Informações, Zope 


\section{INTRODUCTION TO CONTENT MANAGEMENT.}

The article describes the Content Management in Web environment using a compilation of several related texts. It characterizes the CM systems, showing the main tools available and discussing the benefits and risks associated to its implementation. In order to fix the ideas, it joins elements do justify the investment in a CM solution. After the introduction, it proposes a distinction between information, knowledge and content, and suggests that the CM may permit the "Knowlegde Management" in practice. It characterizes the CM tools called CMS and shows the advantages and benefits brought by its implementation into the companies. It also shows an evaluation that permits to calculate the return of the investment (ROI) in a solution of the sort. To follow the Internet evolution as an internal or external communication channel - of relationship of clients, suppliers and partners - the companies need to invest in technologies that permit the distribution and publishing of information in a quick, safe and reliable way. Associated to this evolution, comes the sophistication of the information that must be online, in web pages with images, figures, films and so forth. Changing the way to create and keep web pages, tools called CMS - Content Management Systems - are being created to provide the aspects related above, required by the information treatment in a new stage of the Internet. The CMS tools have resources that permit the content management and these contents may come from different sources and may have different shapes, workflows and treatment schemes. They may be customized and integrated, making easy the use of new mechanisms of querying and permitting the publishing into different equipments like celulars and palms. The benefits brought by the adoption of CMS systems include the reduction of content update costs, the increase of the effectiveness of IT team and the growing of the channels of content publishing. Therefore, it brings new clients and business. Finally, the article shows the challenges such as the stablishment and the standardization of rules to organize contents into repository. Also, the necessity to offer these contents into a standard way, but customized to each client and communication channel. Considering the benefits brought to the possibility of creation, distribution and publishing of contents in a quick, cheap and reliable way, it is expected that the CM may be implemented into the companies in the next years, through the adoption of some CMS tool and behavior change that allow the use of these new resources.

Keywords: : Corporated Portals; Content Management; Knowledge Management; Zope 


\section{INTRODUÇÃO}

O único tipo de conteúdo que existia até pouco tempo nas intranets das empresas em geral eram páginas HTML estáticas, gerenciadas por um webmaster que realizava alterações manuais sempre que necessário. Porém, hoje, os usuários é que ditam quais serão os conteúdos que serão disponibilizados e os tipos destes conteúdos variam muito. Os conteúdos passaram a se originar de fontes diversas, de tipos diversos, e a serem destinados a múltiplos dispositivos.

As páginas HTML estáticas necessitavam de um webmaster e mais ninguém para mantê-las atualizadas no site, uma de cada vez, gerando um gasto de tempo considerável. Hoje, com a maioria dos sites dinâmicos, personalizados, com o conteúdo sendo atualizado diariamente e o grande número de autores produzindo informações dos mais variados tipos, a gerência do conteúdo alterou-se para uma esfera completamente diferente.

As empresas passaram a contar com mais um canal de distribuição de informações para funcionários, clientes, fornecedores e todos os que se relacionam com as mesmas. Passou a ser possível disponibilizar, para toda essa gama de interessados, conteúdos que se sofisticam e crescem em complexidade a cada dia, integrando formatos mais convencionais (textos e figuras) e sons, vídeos, filmes, etc. Neste novo cenário, as empresas passaram a necessitar de ferramentas que permitam cuidar de seu conteúdo informacional de forma ágil.

Segundo CONTENT MANAGEMENT [2001], "Conteúdo é, em essência, qualquer tipo ou unidade de informação digital que é utilizada nas páginas dos websites. Ele pode ser texto, imagem, gráficos, vídeo, som ou, em outras palavras, qualquer coisa que é possível de ser publicada em uma intra, inter ou extranet". A Gestão de Conteúdos, ou "Content Management" apoia organizações na captação, organização e distribuição de conteúdos originários de várias fontes e destinados a diversos tipos de dispositivos de saída.

Este artigo descreve a gestão de conteúdos em ambiente web, a partir do estudo de textos básicos e de informações obtidas em trabalhos relacionados. $\mathrm{O}$ artigo se estrutura como segue: a próxima Seção estabelece uma diferenciação entre informação, conhecimento e conteúdo, sugerindo que a Gestão de Conteúdos permite a Gestão do Conhecimento na prática organizacional. A Seção 3 caracteriza as ferramentas de Gestão de Conteúdos e apresenta vantagens e benefícios advindos de sua implementação nas organizações. Na Seção 4 apresenta-se uma avaliação que permite determinar os retornos sobre investimentos nesse tipo de tecnologia. A Seção 5 conclui fazendo algumas considerações finais.

\section{CONHECIMENTO, CONTEÚDO E INFORMAÇÃO}

Antes de abordar diretamente a Gestão de Conteúdos, é conveniente situá-la de um ponto de vista mais amplo, que permita enxergá-la como nova tecnologia dentro de um conjunto de estudos conhecido como Gestão do Conhecimento. Inicialmente, vejamos uma diferenciação entre os conceitos de informação, conhecimento e conteúdo.

Segundo LEMOS [2001], "Conhecimento sempre significa algum tipo de agregação, algum tipo de adição de valor à informação existente. Um livro numa estante não tem valor até o momento em que alguém se dispõe a lê-lo, a transformar o amontoado de informação que o livro possui”. Ainda segundo LEMOS [2001], "talvez o termo conteúdo esteja mais próximo da idéia de conhecimento 
que a palavra informação". Vale notar que, citando o mesmo autor, "o conteúdo de um website é um conjunto de informações, mas não de qualquer informação. Conteúdo tem um juízo de valor embutido, significa que a informação que o website oferece deve ter coerência, fundamentação, esforço intelectual e operacional dos profissionais que nele trabalham e que agregaram algum tipo de valor à informação”.

O conhecimento, conforme CRUZ [2002], pode ser conceituado como "o entendimento obtido por meio da inferência realizada no contato com dados e informações que traduzam a essência de qualquer elemento. Em outras palavras, conhecimento é saber o quê é e porque é”.

Segundo este mesmo autor, "a Gerência do Conhecimento, ou Knowledge Management (KM), é um conjunto formado por metodologias e tecnologias que têm por finalidade criar condições para identificar, integrar, capturar, recuperar e compartilhar conhecimento existente em qualquer tipo de organização". Pode-se verificar que conhecimento, desse ponto de vista, pressupõe agregar valor através de um processo de transformação realizado sobre dados e informações.

Nessa mesma linha, pode-se afirmar que "conteúdo não é conhecimento, mas pode vir a ser! Conteúdo é tudo que podemos gerenciar em termos de dados e informações e não necessariamente conhecimento" (CRUZ, [2002]).

Na opinião de CRUZ [2002], as organizações precisam definir de forma clara e consistente o conhecimento que precisa ser considerado nos âmbitos operacional, estratégico, e emocional. Esses âmbitos se refletem nos componentes da Gerência do Conhecimento, que são comportamentais, informacionais e tecnológicos. Quando o conteúdo não tem ligação direta com a estratégia de negócios da corporação, ele pode ser classificado apenas como informação. Ao contrário, ao agregarmos valor à informação ou ao conteúdo, ele pode se tornar conhecimento.

Nesse sentido, as ferramentas de CMS ou Gestão de Conteúdos, permitem operacionalizar a Gestão do Conhecimento, fornecendo os mecanismos efetivos de gerenciamento dos conteúdos dos websites para organizações de todo tipo.

Finalmente, segundo CRUZ [2002], "no futuro, essas ferramentas terão capacidade de aprender também e espera-se que até lá já tenhamos aprendido como gerenciar conhecimento". De forma sucinta, pode-se afirmar que as ferramentas de Gestão de Conteúdos são hoje as tecnologias que permitem a implementação da Gestão do Conhecimento na prática das organizações.

\section{A GESTÃO DE CONTEÚDOS}

\section{Portais corporativos}

O Portal Corporativo é o elemento central para a Gestão de Conteúdos. Existe uma distinção entre os conceitos de site, home-page, portal, etc, principalmente em relação ao tamanho dos mesmos. O termo home-page foi o primeiro a ser utilizado na Web, no início dos anos 90 . Através de homepages as pessoas (pesquisadores em universidades) disponibilizavam suas informações em documentos HTML, em geral, com fins pessoais. Um site, ou website, é um conjunto de documentos em linguagem HTML e outras linguagens de scripts, e um portal seria um conjunto maior de sites. 
O site, que anteriormente era encarado como apenas mais um canal de divulgação das organizações, com conteúdos meramente institucionais, sem atualizações sistemáticas e periódicas, tornou-se, no formato de portal corporativo, chave para a implementação da Gestão do Conhecimento nas organizações. Todo o conteúdo que será gerenciado pelas ferramentas CMS estará disponibilizado, através do portal, para empregados, clientes, parceiros e comunidade em geral. A relação entre a Gestão de Conteúdos e os portais corporativos permite estabelecer requisitos fundamentais que garantem a eficiência na sua implementação em uma organização.

Alguns autores se esforçam em classificar os portais por tipos, de acordo com seu conteúdo e funcionalidades. Em geral tais classificações parecem quase sempre arbitrárias. Segundo CRUZ [2002], há cinco tipos de portais, vejamos os dois principais:

- Portal Generalista ou Portal Horizontal. Caracteriza-se por possuir um grande volume de informações coletadas nas mais variadas fontes visando ao atendimento do maior número possível de interesses e necessidades.

- Portal Vertical ou Vortal. Criar cadeias produtivas verticalizadas por tipos de indústria, podendo conter informações sobre os sistemas de produção, com seus diversos processos, desde a obtenção da matéria prima até o produto final.

Hoje as empresas têm que lidar com uma grande quantidade de conteúdos. São informações geradas pelos diferentes departamentos, informações de pesquisa e desenvolvimento, procedimentos técnicos e gerenciais, políticas corporativas, catálogos de produtos e apresentações, press releases, etc.

No apoio à implantação da Gestão do Conhecimento, os Sistemas de Gestão de Conteúdos permitem a criação de portais consistentes em apresentação e conteúdo, com navegação bem planejada, que permite aos autores a possibilidade de compartilhar conhecimento de forma segura e que garante ao usuário atualidade dos conteúdos.

\section{UM MERCADO EM EXPANSÃO}

Segundo pesquisa da ATKERNEY, citada por ANGULO [2001], a quantidade de páginas web publicadas passará de 5 bilhões em 2000 para 40 bilhões em 2003. A Forrester, em pesquisa feita junto a executivos de sites nos Estados Unidos, aponta que $80 \%$ deles irá crescer nos próximos anos, confirmando a previsão de expansão da ATKERNEY. Esta última consultoria estima que 750 milhões de dólares teriam sido desperdiçados em 2001 com ineficiências relacionadas ao atual processo de publicação web.

Esse crescimento veloz e a constatação dos custos de manutenção de um website estático, têm levado organizações a investirem nos chamados CMS ou WCM - Content Management Systems ou Web Content Management. As estimativas de investimentos são da ordem de 1,75 bilhões de dólares em 2002, até 3 bilhões de dólares em 2003 (PERRY, [2001]).

\section{SISTEMAS DE GESTÃO DE CONTEÚDOS (CMS)}

A idéia básica por trás de um CMS é a de separar o gerenciamento do conteúdo do design gráfico das páginas que apresentam o conteúdo. O design das páginas que apresentam os conteúdos são colocados em arquivos chamados moldes (templates), enquanto o conteúdo é armazenado em banco de dados ou arquivos separados. Quando um usuário solicita uma página, as partes são combinadas para produzirem a página HTML padrão. A página resultante pode incluir conteúdos 
de diferentes fontes.

De acordo com PERRY e INFOIMAGEM [2001], o processo de gestão de conteúdos, se divide em três etapas básicas: a criação, a gestão e a publicação.

O CMS deve permitir que os próprios colaboradores, no papel de autores, criem seus conteúdos sem necessidade de intermediários, utilizando os diversos programas disponíveis. Em seguida, estes conteúdos são armazenados em repositórios centralizados para serem tratados (gerenciados, padronizados, formatados e publicados no website) através do CMS. O CMS deve gerir também as revisões, atualizações e o controle de acesso, garantindo confiabilidade ao que será publicado e segurança quanto à propriedade e a autoria dos conteúdos.

Um aspecto importante do CMS é a possibilidade de serem configurados processos simples de controle de fluxos de trabalho, para distribuição dos conteúdos nos canais de comunicação. Ao gerenciar a forma como os conteúdos são publicados, o CMS estabelece fluxos padronizados de trabalho, que definem os ciclos de vida desses conteúdos. Por exemplo, a empresa que anuncia seus produtos na web deverá retirar do site as promoções quando os estoques terminarem, sob pena de se obrigar a continuar vendendo aqueles produtos por preços indevidos.

A inclusão dos conteúdos é controlada pelo uso de marcas (tags) especiais. Estas marcas são em geral proprietárias e exclusivas do CMS usado. Em geral há suporte por linguagens tipo Python, Perl, ou Java para operações mais complexas.

Um bom CMS deve tornar possível time ou colaborarem adicionar, editar e deletar conteúdos. Algumas das funcionalidades básicas cobertas são: esquemas de segurança baseados em papeis, sindicalização de conteúdo, suporte à criação de catálogos, indexação, busca e workflow.

\section{ALGUMAS FERRAMENTAS DISPONÍVEIS}

O termo CMS é usado por uma larga variedade de produtos, produtos comerciais como Vignette e Websphere ou produtos gratuitos como Zope e AOLServer. Tais produtos apresentam uma grande variedade de capacidades, más tendem a incluir os seguintes três componentes básicos:

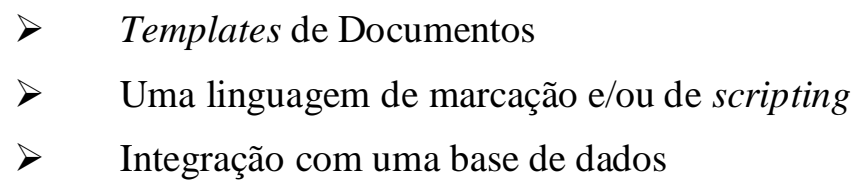

Um incontável número de sistemas e plataformas se anunciam como CMS. CRUZ [2002] os classifica em "ferramentas generalistas" e "ferramentas especialistas". As primeiras servem para coletar, organizar, publicar vários tipos de conteúdos e conhecimentos, permitindo a implantação da Gestão de Conteúdos e de Conhecimento da forma mais abrangente possível. Já as ferramentas especialistas dedicam-se a certos tipos de conteúdos e conhecimentos, como, por exemplo, o gerenciamento de mídias, de redes de computadores e de processos de negócio. A título meramente informativo e ilustrativo, aponta-se, a seguir, alguns desses sistemas.

\section{Zope, uma plataforma de código aberto}


O Zope é um servidor de aplicações ${ }^{1}$ construído por um conjunto de componentes que se integram em torno de um servidor web, um banco de dados e um interpretador que suporta a linguagem Python e scripts que podem ser embebidos em páginas HTML. Zope é escrito em sua maior parte em Python, mas suporta também Perl e uma linguagem de script específica denominada Document Template Markup Language (DTML).

As funcionalidades do Zope podem ser oferecidas, tanto através do seu próprio serviço web, quanto como um processo CGI no Apache, IIS, ou outro servidor web. Isso é um aspecto positivo porque permite que websites pré-existentes possam continuar rodando em outros servidores já instalados que não o servidor web do Zope.

Zope parece ser o servidor de aplicações de código aberto mais referenciado hoje.. Em Zope a atualização, manutenção e administração dos conteúdos nos websites podem ser feitas pela própria web com o navegador, através de uma interface similar a de um gerenciador de arquivos. A maior parte da interação feita com o Zope é realizada através dessa interface completamente baseada na Web. É possível configurar permissões de usuários dando a pessoas níveis de controle diferentes de administração de diferentes seções do site. Nesta interface estão também presentes os controles para se adicionar, deletar, editar páginas, imagens e outros objetos de forma bastante simples. Há também a possibilidade de desfazer (undo) qualquer ação que tenha sido realizada.

Talvez a maior força do Zope seja o fato de tratar páginas web, banco de dados, imagens e quaisquer outros componentes como objetos que podem ser referenciados e reutilizados em todo o site. Zope adota de ponta-a-ponta o conceito de orientação a objetos, utilizando-o de forma a simplificar a gerência de conteúdos. Em Zope as informações são transformadas em objetos, podendo ser dotadas de propriedades e métodos (funções). As noções de modularização, herança e polimorfismo estão presentes. Exatamente como nas linguagens de programação de computadores, a orientação a objetos traz, também para a gestão de conteúdos, maior facilidade ao processo de modelagem de dados e informações, além de maior facilidade de evolução e manutenção dos itens informacionais no portal.

Existe uma variedade enorme de componentes disponíveis desenvolvidos e oferecidos por terceiros no site da comunidade Zope em www.zope.org. Uma vez que os componentes tenham sido baixados e instalados localmente, estes ficam à disposição dos usuários que para utiliza-los simplesmente os adicionam em suas páginas.

\section{Outras plataformas proprietárias}

Vários outras plataformas proprietárias existem hoje no mercado, para citar apenas algumas:

- Documentum, Filenet, Guass, Identitech, Intertech, Lotus Notes - Sistemas de gestão de documentos que começam a oferecer gestão de conteúdos e cujo ponto forte é a existência do "repositório" e na facilidade de acesso.

- Broadvision, Intranet Solutions, Eprise, Vignette, Microsoft Content Management Server - criação, administração, manutenção e publicação de websites - foco especializado na publicação.

- Bentley E-Image - focado nas necessidades especiais da gestão de conteúdos ligados à Engenharia, incluindo suporte a documentos complexos e grandes formatos.

\footnotetext{
${ }^{1}$ Plataforma de software para construção e gerenciamento de aplicações web dinâmicas.
} 
- J.D. Edwards Content Manager - Utiliza o Microsoft Office para gerir e reutilizar documentos nas empresas, permitindo o compartilhamento de componentes de conteúdo em múltiplos formatos, facilitando o desenvolvimento e a publicação de novos conteúdos.

\section{BENEFÍCIOS DE UM CMS}

Um CMS oferece benefícios para ambos administradores e autores. Talvez o maior deles é a possibilidade de usar templates e elementos comuns de design que asseguram a consistência de apresentação do site como um todo. A responsabilidade pela aparência das páginas web é retirada dos autores e centralizada. Isso simplifica a manutenção do site.

Autores incorporam os templates em seus documentos adicionando uma pequena quantidade de código extra. Assim eles podem se concentrar no conteúdo ao invés de terem que se preocupar também com a apresentação. Para alterar a aparência do site, os administradores precisam apenas modificar os templates e não cada uma das páginas individualmente.

Um CMS simplifica também a delegação de responsabilidades pela provisão e manutenção do conteúdo do portal. Um CMS possibilita, em geral, níveis diferentes de acesso serem configurados para separar áreas do portal sem que o responsável pela configuração tenha que descer ao nível de controle de permissão do sistema operacional. Em geral essa operação pode ser feita através da interface do navegador.

Funcionalidades extras, tais como mecanismos de busca, calendários, webmail, e outros componentes podem ser construídos no próprio ambiente, ou disponibilizados por terceiros como plug-ins. A provisão de funcionalidades economiza tempo que seria normalmente gasto no desenvolvimento ou tentativa de integrar tais componentes. CMS's mais avançados oferecem benefícios adicionais de segurança, ferramentas de gerenciamento, facilitam a interação com a base de dados, além de ferramentas de indexação e catálogo. Estes benefícios reduzem o tempo de desenvolvimento de outros componentes do portal e garantem a sua evolução.

\section{PORQUE USAR UM CMS}

A implantação de um CMS ajuda as empresas a:

- $\quad$ padronizar as estruturas de conteúdo e manutenção do controle de apresentação e uso da marca em toda a organização;

- aumentar a eficiência das equipes: usuários (autores) publicam suas informações e técnicos (administradores) cuidam da infra-estrutura;

- agregar valor aos conteúdos web à partir do estabelecimento de fluxos de trabalho com processo de aprovação padronizado;

- $\quad$ reduzir custos de atualização de seus conteúdos e aumentar a velocidade e frequiência da publicação de novas informações;

- reduzir custos de criação/manutenção/evolução disponibilizando componentes préfabricados e processos pré-automatizados.

Além disso, ao permitir que os próprios autores façam, de forma adequada e segura, a atualização de seus conteúdos, diminui-se a carga de trabalho da equipe de TI.

Segundo MICROSOFT [2001], inúmeros custos relacionados à manutenção dos portais podem ser reduzidos com o uso de CMS, para citar alguns: 
- Criação de conteúdos originais e conversão desses originais em conteúdo apropriado para publicação web;

- Revisão prévia do conteúdo antes da publicação;

- Correções relacionadas à publicações incorretas ou inadequadas, como preços errados ou falta de apoio para navegação ou para obtenção de novas informações;

- Desatualização dos conteúdos, prejudicando a tomada de decisões;

- $\quad$ Tempo gasto para filtragem de conteúdos irrelevantes, fazendo com que os clientes abandonem o portal;

- $\quad$ Falta de uma padronização no uso da marca e do estilo da organização;

- Manutenção geral do sistema, incluindo o gerenciamento da segurança, da escalabilidade e da política de direitos de usuários e regras de uso;

- $\quad$ Atualização das aplicações Web em todas as partes das organizações;

Pode-se dizer que a chave para obtenção de retorno sobre o investimento em ferramentas para gestão de conteúdos inclui escolher a ferramenta adequada para as necessidades do negócio e assegurar-se da rápida implementação e desenvolvimento da ferramenta selecionada e adquirida. Para isso, o CMS deve ser de fácil assimilação e deve permitir às equipes de colaboração o seu rápido aprendizado.

\section{VALE A PENA INVESTIR EM GESTÃO DE CONTEÚDOS?}

Para se avaliar o retorno sobre o investimento em um CMS, as organizações devem avaliar as melhorias que podem ser fornecidas pelo sistema, discriminando todos os fatores possíveis, como tempo de resposta, atualização das informações e todos os demais parâmetros cuja melhoria representa ganho para a organização como um todo. Em seguida, a organização deve avaliar a chance de o sistema ser realmente adotado e utilizado.

A idéia é apurar todos os ganhos trazidos pelo CMS, do ponto de vista operacional e estratégico, nos processos das organizações e compará-los com o custo do investimento, o qual leva em consideração, não apenas a compra da ferramenta, mas também os custos de treinamento de pessoal, o planejamento para implantação e a implementação em si.

Assim, segundo MICROSOFT [2001], o retorno sobre o investimento em um CMS poderia ser definido como a soma das economias de custos (EC), do aumento do valor dos conteúdos (AVC) e da inovação (I), multiplicada pela probabilidade de adoção do CMS (PA):

$$
\text { Retorno }=(\mathrm{EC}+\mathrm{AVC}+\mathrm{I}) * \mathrm{PA}
$$

Onde,

$$
\begin{aligned}
\text { Economia de custos }(\mathrm{EC})=\text { padronização } & + \text { utilização de recursos } \\
& + \text { custo de atualização } \\
& + \text { tempo de recuperação }
\end{aligned}
$$

Aumento valor dos conteúdos $(\mathrm{AVC})=$ valor da mensagem $*$ relevância

$$
\begin{aligned}
& \text { * oportunidade } \\
& \text { * precisão } \\
& \text { * flexibilidade }
\end{aligned}
$$


Inovação $(\mathrm{I})=$ flexibilidade da ferramenta de desenvolvimento + compatibilidade para integração *

redução do tempo para distribuição

\author{
Probabilidade de adoção $(\mathrm{PA})=$ compatibilidade da $\mathrm{TI} *$ \\ flexibilidade para vários tipos de negócios / \\ usuários * escalabilidade
}
Investimento $=$ número de usuários *
(mudanças de processos + custos de treinamento ) +
custo do sistema CMS + custo do projeto de implementação

\title{
CONCLUSÕES
}

A Gestão de Conteúdos é uma abordagem tecnológica que surge em função da explosão de conteúdos multimídia na web e em intranets e visa permitir a gerência de todas as etapas, desde a criação até a publicação de conteúdos, de forma padronizada, trazendo redução de custos e facilitando o intercâmbio de conteúdos entre portais.

Há uma série de benefícios e desafios ligados a adoção de um CMS pelas empresas. Os benefícios incluem a redução de custos de manutenção de portais e o aumento dos canais de publicação dos conteúdos, trazendo clientes e negócios. Os desafios vão desde a definição e padronização de regras para colocação de informações em "repositórios" bem como no estabelecimento de possibilidades de consultas até a necessidade de se disponibilizar o conteúdo informacional de forma customizada para cada cliente e tipo de canal de publicação.

Os aspectos mais importantes da Gestão de Conteúdos podem ser agrupados nas categorias técnica e comportamental. Do ponto de vista técnico, pode-se dizer que a Gestão de Conteúdos é uma tecnologia emergente que será utilizada pela maioria das corporações para dar conta do volume de informações e dados que precisarão ser contextualizados e compartilhados através dos mais diferentes dispositivos.

Do ponto de vista comportamental ou cultural, ao abraçar todos os processos das organizações, disponibilizar seu "conhecimento" para os parceiros, incluir aspectos de gestão de documentos e de processos de trabalho, a Gestão de Conteúdos sugere uma mudança cultural profunda. Sem ela, toda a tecnologia não convergirá num conjunto que possa funcionar de forma integrada.

Assim a implementação e os benefícios da Gestão de Conteúdos estão intimamente ligados ao aspecto humano, o que faz com que todo o planejamento estratégico da Gestão de Conteúdos não possa prescindir da mudança de comportamento das pessoas, criando um ambiente propício para o 
desenvolvimento, compartilhamento e uso de conhecimento dentro da organização.

\section{REFERÊNCIAS BIBLIOGRÁFICAS}

ANGULO, Marcelo Junqueira. Porque gerenciar conteúdos. 2002. Disponível em: <http://www.hiperlogica.com.br> Acesso em: 18 mar. 2002.

CONTENT MANAGEMENT. 2001. Disponível em <http://www.contentmanager.eu.com> Acesso em: 18 mar. 2002.

CRUZ, Tadeu. Gerência do Conhecimento. Ed. Cobra, São Paulo, 2002.

INFOIMAGEM. Gestão de quê, Documentos? Conteúdos? Conhecimento? Jornal da Gestão Electrônica de Imagens, Documentos e Processos - maio/junho 2001. Disponível em: <http://www.doc-imagem.com/infoimagem/info33/33art1.htm> Acesso em: 18 mar. 2002.

J.D. EDWARS. Notícias. 2001. Disponível em 〈http://www.jdedwars.com.pt $>$ Acesso em: 18 mar. 2002.

LEMOS, Paulo. Conteúdo: quem faz, como faz. Junho, 2000, Brasil Investiment Link. Disponível

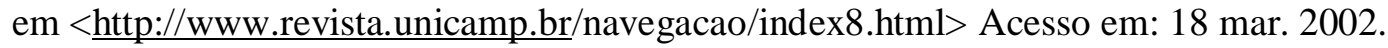

MICROSOFT. The Business Case for a Web content management System. 2001. Disponível em: <http://www.microsoft.com/cmserver> Acesso em: 18 mar. 2002.

PERRY, Robert. Managing the Content Explosion into Content-Rich Applications. Internet Computing Strategies Report, YANKEE GROUP REPORT, Vol. 6, No. 2, May 2001.

STONEBRAKER, M. \& HELLERSTEIN, J.M. Content Integration for E-Business. 2001, Cohera Corporation. Disponível em: 〈http://www.cohera.com> Acesso em: 18 mar. 2002.

ZOPE. Z objetc publishing environment. Disponível em <http://www.zope.com>. Acesso em: 18 mar. 2002.

WELCHMAN, Lisa. Effective Web Content Management: Different Sites, Different Methods. June 2001. Disponível em <http://www.webcms.org>. Acesso em: 18 mar. 2002. 